\title{
ON THE $x$-COORDINATES OF PELL EQUATIONS THAT ARE PRODUCTS OF TWO LUCAS NUMBERS
}

\author{
MAHADI DDAMULIRA
}

\begin{abstract}
Let $\left\{L_{n}\right\}_{n \geq 0}$ be the sequence of Lucas numbers given by $L_{0}=2, L_{1}=1$ and $L_{n+2}=L_{n+1}+L_{n}$ for all $n \geq 0$. In this paper, for an integer $d \geq 2$ which is square-free, we show that there is at most one value of the positive integer $x$ participating in the Pell equation $x^{2}-d y^{2}= \pm 1$ which is a product of two Lucas numbers, with a few exceptions that we completely characterize.
\end{abstract}

\section{INTRODUCTION}

Let $\left\{L_{n}\right\}_{n \geq 0}$ be the sequence of Lucas numbers given by $L_{0}=2, L_{1}=1$ and

$$
L_{n+2}=L_{n+1}+L_{n}
$$

for all $n \geq 0$. This is sequence A000032 on the On-Line Encyclopedia of Integer Sequences (OEIS) [19]. The first few terms of this sequence are

$$
\left\{L_{n}\right\}_{n \geq 0}=2,1,3,4,7,11,18,29,47,76,123,199,322,521,843,1364,2207,3571, \ldots
$$

Putting $(\alpha, \beta)=\left(\frac{1+\sqrt{5}}{2}, \frac{1-\sqrt{5}}{2}\right)$ for the roots of the characteristic equation $r^{2}-r-1=0$ of the Lucas sequence, the Binet formula for its general terms is given by

$$
L_{n}=\alpha^{n}+\beta^{n}, \quad \text { for all } \quad n \geq 0 .
$$

Furthermore, we can prove by induction that the inequality

$$
\alpha^{n-1} \leq L_{n} \leq \alpha^{n+2},
$$

holds for all $n \geq 0$.

Let $d \geq 2$ be a positive integer which is not a perfect square. It is well-known that the Pell equation

$$
x^{2}-d y^{2}= \pm 1
$$

has infinitely many positive integer solutions $(x, y)$. Letting $\left(x_{1}, y_{1}\right)$ be the smallest positive solution, all solutions are of the form $\left(x_{k}, y_{k}\right)$ for some positive integer $k$, where

$$
x_{k}+y_{k} \sqrt{d}=\left(x_{1}+y_{1} \sqrt{d}\right)^{k} \quad \text { for all } \quad k \geq 1 .
$$

Furthermore, the sequence $\left\{x_{k}\right\}_{k \geq 1}$ is binary recurrent. The following formula

$$
x_{k}=\frac{\left(x_{1}+y_{1} \sqrt{d}\right)^{k}+\left(x_{1}-y_{1} \sqrt{d}\right)^{k}}{2},
$$

holds for all positive integers $k$.

Kafle, et al. [11] considered the Diophantine equation

$$
x_{n}=F_{\ell} F_{m}
$$

FEBRUARY 2020 


\section{DDAMULIRA}

where $\left\{F_{m}\right\}_{m \geq 0}$ is the sequence of Fibonacci numbers given by $F_{0}=0, F_{1}=1$ and $F_{m+2}=$ $F_{m+1}+F_{m}$ for all $m \geq 0$. They proved that equation (1.5) has at most one solution $n$ in positive integers except for $d=2,3,5$, for which case equation (1.5) has the solutions $x_{1}=1$ and $x_{2}=3, x_{1}=2$ and $x_{2}=26, x_{1}=2$ and $x_{2}=9$, respectively.

There are many other researchers who have studied related problems involving the intersection sequence $\left\{x_{n}\right\}_{n \geq 1}$ with linear recurrence sequences of interest. For example, see $[4,8,7,9,12,13,14,16,17,20]$.

\section{Main Result}

In this paper, we study a similar problem to that of Kafle, et al. [11], but with the Lucas numbers instead of the Fibonacci numbers. That is, we show that there is at most one value of the positive integer $x$ participating in (1.3), which is a product of two Lucas numbers, with a few exceptions that we completely characterize. This can be interpreted as solving the Diophantine equation

$$
x_{k}=L_{n} L_{m}
$$

in nonnegative integers $(k, n, m)$ with $k \geq 1$ and $0 \leq m \leq n$.

Theorem 2.1. For each square-free integer $d \geq 2$, there is at most one integer $k$ such that the equation (2.1) holds, except for $d \in\{2,3,5,15,17,35\}$ for which $x_{1}=1, x_{2}=3, x_{3}=7, x_{9}=$ 1393 (for $d=2), x_{1}=2, x_{2}=7$ (for $\left.d=3\right), x_{1}=2, x_{2}=9$ (for $\left.d=5\right), x_{1}=4, x_{5}=15124$ (for $d=15), x_{1}=4, x_{2}=33($ for $d=17)$, and $x_{1}=6, x_{3}=846($ for $d=35)$.

\section{Preliminary Results}

3.1. Notations and terminology from algebraic number theory. We begin by recalling some basic notions from algebraic number theory.

Let $\eta$ be an algebraic number of degree $d$ with minimal primitive polynomial over the integers

$$
a_{0} x^{d}+a_{1} x^{d-1}+\cdots+a_{d}=a_{0} \prod_{i=1}^{d}\left(x-\eta^{(i)}\right),
$$

where the leading coefficient $a_{0}$ is positive and the $\eta^{(i)}$ 's are the conjugates of $\eta$. Then, the logarithmic height of $\eta$ is given by

$$
h(\eta):=\frac{1}{d}\left(\log a_{0}+\sum_{i=1}^{d} \log \left(\max \left\{\left|\eta^{(i)}\right|, 1\right\}\right)\right) .
$$

In particular, if $\eta=p / q$ is a rational number with $\operatorname{gcd}(p, q)=1$ and $q>0$, then $h(\eta)=$ $\log \max \{|p|, q\}$. The following are some of the properties of the logarithmic height function $h(\cdot)$, which will be used in the next sections of this paper without reference:

$$
\begin{aligned}
h(\eta \pm \gamma) & \leq h(\eta)+h(\gamma)+\log 2 \\
h\left(\eta \gamma^{ \pm 1}\right) & \leq h(\eta)+h(\gamma), \\
h\left(\eta^{s}\right) & =|s| h(\eta) \quad(s \in \mathbb{Z}) .
\end{aligned}
$$


3.2. Linear forms in logarithms. In order to prove our main result Theorem 2.1, we need to use several times a Baker-type lower bound for a nonzero linear form in logarithms of algebraic numbers. There are many such in the literature like that of Baker and Wüstholz from [2]. We start by recalling the result of Bugeaud, Mignotte, and Siksek ([5], Theorem 9.4, pp. 989), which is a modified version of the result of Matveev [18], which is one of our main tools in this paper.

Theorem 3.1. Let $\gamma_{1}, \ldots, \gamma_{t}$ be positive real numbers in a number field $\mathbb{K} \subseteq \mathbb{R}$ of degree $D$, $b_{1}, \ldots, b_{t}$ be nonzero integers, and assume that

$$
\Lambda:=\gamma_{1}^{b_{1}} \cdots \gamma_{t}^{b_{t}}-1
$$

is nonzero. Then,

$$
\log |\Lambda|>-1.4 \times 30^{t+3} \times t^{4.5} \times D^{2}(1+\log D)(1+\log B) A_{1} \cdots A_{t},
$$

where

$$
B \geq \max \left\{\left|b_{1}\right|, \ldots,\left|b_{t}\right|\right\}
$$

and

$$
A_{i} \geq \max \left\{D h\left(\gamma_{i}\right),\left|\log \gamma_{i}\right|, 0.16\right\}, \quad \text { for all } \quad i=1, \ldots, t .
$$

When $t=2$ and $\gamma_{1}$ and $\gamma_{2}$ are positive and multiplicatively independent, we can use a result of Laurent, Mignotte, and Nesterenko [15]. Namely, in this case let $B_{1}$ and $B_{2}$ be real numbers larger than 1 such that

$$
\log B_{i} \geq \max \left\{h\left(\gamma_{i}\right), \frac{\left|\log \gamma_{i}\right|}{D}, \frac{1}{D}\right\}, \quad \text { for } \quad i=1,2
$$

and put

$$
b^{\prime}=\frac{\left|b_{1}\right|}{D \log B_{2}}+\frac{\left|b_{2}\right|}{D \log B_{1}} .
$$

Put

$$
\Gamma=b_{1} \log \gamma_{1}+b_{2} \log \gamma_{2} .
$$

We note that $\Gamma \neq 0$ because $\gamma_{1}$ and $\gamma_{2}$ are multiplicatively independent. The following result is Corollary 2 in [15].

Theorem 3.2. With the above notations, assuming that $\gamma_{1}$ and $\gamma_{2}$ are positive and multiplicatively independent, then

$$
\log |\Gamma|>-24.34 D^{4}\left(\max \left\{\log b^{\prime}+0.14, \frac{21}{D}, \frac{1}{2}\right\}\right)^{2} \log B_{1} \log B_{2} .
$$

Note that with $\Gamma$ given by (3.3), we have $e^{\Gamma}-1=\Lambda$, where $\Lambda$ is given by (3.2) in case $t=2$, which explains the connection between Theorem 3.1 and Theorem 3.2.

3.3. Reduction procedure. During the calculations, we get upper bounds on our variables which are too large, thus we need to reduce them. To do so, we use some results from the theory of continued fractions.

For the treatment of linear forms homogeneous in two integer variables, we use the wellknown classic result in the theory of Diophantine approximation. 


\section{DDAMULIRA}

Lemma 3.3. Let $\tau$ be an irrational number, $\frac{p_{0}}{q_{0}}, \frac{p_{1}}{q_{1}}, \frac{p_{2}}{q_{2}}, \ldots$ be all the convergents of the continued fraction of $\tau$, and $M$ be a positive integer. Let $N$ be a nonnegative integer such that $q_{N}>M$. Then, putting $a(M):=\max \left\{a_{i}: i=0,1,2, \ldots, N\right\}$, the inequality

$$
\left|\tau-\frac{r}{s}\right|>\frac{1}{(a(M)+2) s^{2}}
$$

holds for all pairs $(r, s)$ of positive integers with $0<s<M$.

For a nonhomogeneous linear form in two integer variables, we use a slight variation of a result due to Dujella and Pethő (see [10], Lemma 5a). For a real number $X$, we write $\|X\|=\min \{|X-n|: n \in \mathbb{Z}\}$ for the distance from $X$ to the nearest integer.

Lemma 3.4. Let $M$ be a positive integer, $\frac{p}{q}$ be a convergent of the continued fraction of the irrational number $\tau$ such that $q>6 M$, and $A, B, \mu$ be some real numbers with $A>0$ and $B>1$. Let further $\varepsilon=\|\mu q\|-M\|\tau q\|$. If $\varepsilon>0$, then there is no solution to the inequality

$$
0<|u \tau-v+\mu|<A B^{-w},
$$

in positive integers $u, v$, and $w$ with

$$
u \leq M \quad \text { and } \quad w \geq \frac{\log (A q / \varepsilon)}{\log B} .
$$

At various occasions, we need to find a lower bound for linear forms in logarithms with bounded integer coefficients in three and four variables. In this case, we use the LLL algorithm that we describe below. Let $\tau_{1}, \tau_{2}, \ldots \tau_{t} \in \mathbb{R}$ and the linear form

$$
x_{1} \tau_{1}+x_{2} \tau_{2}+\cdots+x_{t} \tau_{t} \quad \text { with } \quad\left|x_{i}\right| \leq X_{i} .
$$

We put $X=\max \left\{X_{i}\right\}, C>(t X)^{t}$ and consider the integer lattice $\Omega$ generated by

$$
\mathbf{b}_{j}=\mathbf{e}_{j}+\left\lfloor C \tau_{j}\right\rceil \text { for } \quad 1 \leq j \leq t-1 \quad \text { and } \quad \mathbf{b}_{t}=\left\lfloor C \tau_{t}\right\rceil \mathbf{e}_{t},
$$

where $C$ is a sufficiently large positive constant.

Lemma 3.5. Let $X_{1}, X_{2}, \ldots, X_{t}$ be positive integers such that $X:=\max \left\{X_{i}\right\}$ and $C>(t X)^{t}$ is a fixed sufficiently large constant. With the above notation on the lattice $\Omega$, we consider a reduced base $\left\{\boldsymbol{b}_{i}\right\}$ to $\Omega$ and its associated Gram-Schmidt orthogonalization base $\left\{\boldsymbol{b}_{i}^{*}\right\}$. We set

$$
c_{1}=\max _{1 \leq i \leq t} \frac{\left\|\boldsymbol{b}_{1}\right\|}{\left\|\boldsymbol{b}_{i}^{*}\right\|}, \quad \theta=\frac{\left\|\boldsymbol{b}_{1}\right\|}{c_{1}}, \quad Q=\sum_{i=1}^{t-1} X_{i}^{2}, \quad \text { and } \quad R=\left(1+\sum_{i=1}^{t} X_{i}\right) / 2 .
$$

If the integers $x_{i}$ are such that $\left|x_{i}\right| \leq X_{i}$, for $1 \leq i \leq t$ and $\theta^{2} \geq Q+R^{2}$, then we have

$$
\left|\sum_{i=1}^{t} x_{i} \tau_{i}\right| \geq \frac{\sqrt{\theta^{2}-Q}-R}{C}
$$

For the proof and further details, we refer the reader to the book of Cohen. (Proposition 2.3.20 in [6], pp. 58-63). 
3.4. Pell equations and Dickson polynomials. Here we give some relations about Pell equations and Dickson polynomials that will be useful in the next section of this paper.

Let $d \geq 2$ be a squarefree integer. We put $\delta=x_{1}+\sqrt{x_{1}^{2}-\epsilon}$ for the smallest positive integer $x_{1}$ such that

$$
x_{1}^{2}-d y_{1}^{2}=\epsilon, \quad \epsilon \in\{ \pm 1\}
$$

for some positive integer $y_{1}$. Then,

$$
x_{k}+y_{k} \sqrt{d}=\delta^{k} \quad \text { and } \quad x_{k}-y_{k} \sqrt{d}=\eta^{k}, \quad \text { where } \quad \eta:=\epsilon \delta^{-1} .
$$

From the above, we get

$$
2 x_{k}=\delta^{k}+\left(\epsilon \delta^{-1}\right)^{k} \quad \text { for all } \quad k \geq 1 .
$$

There is a formula expressing $2 x_{k}$ in terms of $2 x_{1}$ by means of the Dickson polynomial $D_{k}\left(2 x_{1}, \epsilon\right)$, where

$$
D_{k}(x, y)=\sum_{i=0}^{\lfloor k / 2\rfloor} \frac{k}{k-i}\left(\begin{array}{c}
k-i \\
i
\end{array}\right)(-y)^{i} x^{k-2 i}
$$

These polynomials appear naturally in many number theory problems and results, for example in a result of Bilu and Tichy [3] concerning polynomials $f(X), g(X) \in \mathbb{Z}[X]$ such that the Diophantine equation $f(x)=g(y)$ has infinitely many integer solutions $(x, y)$.

Example 3.6. $\quad$ (i) $k=2$. We have

$$
2 x_{2}=\sum_{i=0}^{1} \frac{2}{2-i}\left(\begin{array}{c}
2-i \\
i
\end{array}\right)(-\epsilon)^{i}\left(2 x_{1}\right)^{2-2 i}=4 x_{1}^{2}-2 \epsilon, \quad \text { so } \quad x_{2}=2 x_{1}^{2}-\epsilon .
$$

(ii) $k=3$. We have

$$
2 x_{3}=\sum_{i=0}^{1} \frac{3}{3-i}\left(\begin{array}{c}
3-i \\
i
\end{array}\right)(-\epsilon)^{i}\left(2 x_{1}\right)^{3-2 i}=\left(2 x_{1}\right)^{3}-3 \epsilon\left(2 x_{1}\right), \quad \text { so } \quad x_{3}=4 x_{1}^{3}-3 \epsilon x_{1} .
$$

\section{Bounding THE VARIABLES}

We assume that $\left(x_{1}, y_{1}\right)$ is the smallest positive solution of the Pell equation (1.3). As in Subsection 3.4, we set

and put

$$
x_{1}^{2}-d y_{1}^{2}=\epsilon, \quad \epsilon \in\{ \pm 1\},
$$

$$
\delta=x_{1}+\sqrt{d} y_{1} \quad \text { and } \quad \eta=x_{1}-\sqrt{d} y_{1}=\epsilon \delta^{-1} .
$$

From (1.4), we get

$$
x_{k}=\frac{1}{2}\left(\delta^{k}+\eta^{k}\right) .
$$

Since $\delta \geq 1+\sqrt{2}>\alpha^{3 / 2}$, it follows that the estimate

$$
\frac{\delta^{k}}{\alpha^{2}} \leq x_{k}<\frac{\delta^{k}}{\alpha} \quad \text { holds for all } k \geq 1 .
$$

We let $(k, n, m):=\left(k_{i}, n_{i}, m_{i}\right)$ for $i=1,2$ be the solutions of (2.1). By (1.2) and (4.2), we get

so

$$
\alpha^{n+m-2} \leq L_{n} L_{m}=x_{k}<\frac{\delta^{k}}{\alpha} \quad \text { and } \quad \frac{\delta^{k}}{\alpha^{2}} \leq x_{k}=L_{n} L_{m} \leq \alpha^{n+m+4},
$$

$$
k c_{1} \log \delta-6<n+m<k c_{1} \log \delta+1 \quad \text { where } \quad c_{1}=\frac{1}{\log \alpha} .
$$




\section{DDAMULIRA}

To fix ideas, we assume that

$$
n \geq m \quad \text { and } \quad k_{1}<k_{2} \text {. }
$$

We also put

$$
m_{3}=\min \left\{m_{1}, m_{2}\right\}, \quad m_{4}=\max \left\{m_{1}, m_{2}\right\}, \quad n_{3}=\min \left\{n_{1}, n_{2}\right\}, \quad n_{4}=\max \left\{n_{1}, n_{2}\right\} .
$$

Using the inequality (4.4) together with the fact that $\delta \geq 1+\sqrt{2}=\alpha^{3 / 2}$ (so, $c_{1} \log \delta>3 / 2$ ), gives us that

$$
\frac{3}{2} k_{2}<k_{2} c_{1} \log \delta<2 n_{2}+6 \leq 2 n_{4}+6
$$

so

$$
k_{1}<k_{2}<\frac{4}{3} n_{4}+4
$$

Thus, it is enough to find an upper bound on $n_{4}$. Substituting (1.1) and (4.1) in (2.1) we get

$$
\frac{1}{2}\left(\delta^{k}+\eta^{k}\right)=\left(\alpha^{n}+\beta^{n}\right)\left(\alpha^{m}+\beta^{m}\right) .
$$

This can be regrouped as

$$
\delta^{k} 2^{-1} \alpha^{-n-m}-1=-2^{-1} \eta^{k} \alpha^{-n-m}+\left(\beta \alpha^{-1}\right)^{n}+\left(\beta \alpha^{-1}\right)^{m}+\left(\beta \alpha^{-1}\right)^{n+m} .
$$

Since $\beta=-\alpha^{-1}, \eta=\varepsilon \delta^{-1}$ and using the fact that $\delta^{k} \geq \alpha^{n+m-1}$ (by (4.3)), we get

$$
\begin{aligned}
\left|\delta^{k} 2^{-1} \alpha^{-n-m}-1\right| & \leq \frac{1}{2 \delta^{k} \alpha^{n+m}}+\frac{1}{\alpha^{2 n}}+\frac{1}{\alpha^{2 m}}+\frac{1}{\alpha^{2(n+m)}} \\
& \leq \frac{\alpha}{2 \alpha^{2(n+m)}}+\frac{3}{\alpha^{2 m}}<\frac{6}{\alpha^{2 m}},
\end{aligned}
$$

In the above, we have also used the facts that $n \geq m$ and $(1 / 2) \alpha+3<6$. Hence,

$$
\left|\delta^{k} 2^{-1} \alpha^{-n-m}-1\right|<\frac{6}{\alpha^{2 m}} \text {. }
$$

We let $\Lambda_{1}=\delta^{k} 2^{-1} \alpha^{-n-m}-1$. We put

$$
\Gamma_{1}=k \log \delta-\log 2-(n+m) \log \alpha .
$$

Note that $e^{\Gamma_{1}}-1=\Lambda_{1}$. If $m>100$, then $\frac{6}{\alpha^{2 m}}<\frac{1}{2}$. Since $\left|e^{\Gamma_{1}}-1\right|<1 / 2$, it follows that

$$
\left|\Gamma_{1}\right|<2\left|e^{\Gamma_{1}}-1\right|<\frac{12}{\alpha^{2 m}} .
$$

By recalling that $(k, n, m)=\left(k_{i}, n_{i}, m_{i}\right)$ for $i=1,2$, we get that

$$
\left|k_{i} \log \delta-\log 2-\left(n_{i}+m_{i}\right) \log \alpha\right|<\frac{12}{\alpha^{2 m_{i}}}
$$

holds for both $i=1,2$ provided $m_{3}>100$.

We apply Theorem 3.1 on the left-hand side of (4.7). First, we need to check that $\Lambda_{1} \neq 0$. If it were, then $\delta^{k} \alpha^{-n-m}=2$. However, this is impossible since $\delta^{k} \alpha^{-n-m}$ is a unit, whereas 2 is not. Thus, $\Lambda_{1} \neq 0$, and we can apply Theorem 3.1. We take the data

$$
t=3, \quad \gamma_{1}=\delta, \quad \gamma_{2}=2, \quad \gamma_{3}=\alpha, \quad b_{1}=k, \quad b_{2}=-1, \quad b_{3}=-n-m .
$$

We take $\mathbb{K}=\mathbb{Q}(\sqrt{d}, \alpha)$ which has degree $D \leq 4$ (it could be that $d=5$ in which case $D=2$; otherwise, $D=4$ ). Since $\delta \geq 1+\sqrt{2}>\alpha$, the second inequality in (4.4) tells us that $k<n+m$, so we take $B=2 n$. We have $h\left(\gamma_{1}\right)=h(\delta)=\frac{1}{2} \log \delta, h\left(\gamma_{2}\right)=h(2)=\log 2$, and 
$h\left(\gamma_{3}\right)=h(\alpha)=\frac{1}{2} \log \alpha$. Thus, we can take $A_{1}=2 \log \delta, A_{2}=4 \log 2$, and $A_{3}=2 \log \alpha$. Now, Theorem 3.1 tells us that

$$
\begin{aligned}
\log \left|\Lambda_{1}\right| & >-1.4 \times 30^{6} \times 3^{4.5} \times 4^{2}(1+\log 4)(1+\log (2 n))(2 \log \delta)(4 \log 2)(2 \log \alpha) \\
& >-2.92 \times 10^{13} \log \delta(1+\log (2 n)) .
\end{aligned}
$$

By comparing the above inequality with (4.7), we get

$$
2 m \log \alpha-\log 6<2.92 \times 10^{13} \log \delta(1+\log (2 n)) .
$$

Thus,

$$
m<6.06 \times 10^{13} \log \delta(1+\log (2 n)) .
$$

Since, $\delta^{k}<\alpha^{n+m+6}$, we get that

$$
k \log \delta<(n+m+6) \log \alpha \leq(2 n+6) \log \alpha,
$$

which together with the estimate (4.12) gives

$$
k m<5.84 \times 10^{13} n(1+\log (2 n)) .
$$

We have just proved the following lemma, which will be important later.

Lemma 4.1. If $x_{k}=L_{n} L_{m}$ and $n \geq m$, then

$m<6.06 \times 10^{13} \log \delta(1+\log (2 n)), \quad k m<5.84 \times 10^{13} n(1+\log (2 n)), \quad k \log \delta<4 n \log \alpha$.

Note that we did not assume that $m_{3}>100$ for Lemma 4.1 because we have worked with the inequality (4.7) and not (4.9). We again assume that $m_{3}>100$. Then, the two inequalities (4.10) hold. We eliminate the term involving $\log \delta$ by multiplying the inequality for $i=1$ with $k_{2}$ and the one for $i=2$ with $k_{1}$, subtract them, and apply the triangle inequality as follows

$$
\begin{aligned}
& \left|\left(k_{2}-k_{1}\right) \log 2-\left(k_{2}\left(n_{1}+m_{1}\right)-k_{1}\left(n_{2}+m_{2}\right)\right) \log \alpha\right| \\
& =\left|k_{2}\left(k_{1} \log \delta-\log 2-\left(n_{1}+m_{1}\right) \log \alpha\right)-k_{1}\left(k_{2} \log \delta-\log 2-\left(n_{2}+m_{2}\right) \log \alpha\right)\right| \\
& \leq k_{2}\left|k_{1} \log \delta-\log 2-\left(n_{1}+m_{1}\right) \log \alpha\right|+k_{1}\left|k_{2} \log \delta-\log 2-\left(n_{2}+m_{2}\right) \log \alpha\right| \\
& \leq \frac{12 k_{2}}{\alpha^{2 m_{1}}}+\frac{12 k_{1}}{\alpha^{2 k_{2}}}<\frac{24 k_{2}}{\alpha^{2 m_{3}}} .
\end{aligned}
$$

Thus,

$$
\left|\Gamma_{2}\right|:=\left|\left(k_{2}-k_{1}\right) \log 2-\left(k_{2}\left(n_{1}+m_{1}\right)-k_{1}\left(n_{2}+m_{2}\right)\right) \log \alpha\right|<\frac{24 k_{2}}{\alpha^{2 m_{3}}} .
$$

We are now set to apply Theorem 3.2 with the data

$$
t=2, \quad \gamma_{1}=2, \quad \gamma_{2}=\alpha, \quad b_{1}=k_{2}-k_{1}, \quad b_{2}=k_{2}\left(n_{1}+m_{1}\right)-k_{1}\left(n_{2}+m_{2}\right) .
$$

That $\gamma_{1}=2$ and $\gamma_{2}=\alpha$ are multiplicatively independent follows because $\alpha$ is a unit whereas 2 is not. We observe that $k_{2}-k_{1}<k_{2}$, whereas by the absolute value of the inequality in (4.15), we have

$$
\left|k_{2}\left(n_{1}+m_{1}\right)-k_{1}\left(n_{2}+m_{2}\right)\right| \leq\left(k_{2}-k_{1}\right) \frac{\log 2}{\log \alpha}+\frac{24 k_{2}}{\alpha^{2 m_{3}} \log \alpha}<2 k_{2},
$$

because $m_{3}>10$. We have that $\mathbb{K}=\mathbb{Q}(\alpha)$, which has $D=2$. So, we can take

$$
\log B_{1}=\max \left\{h\left(\gamma_{1}\right), \frac{\left|\log \gamma_{1}\right|}{2}, \frac{1}{2}\right\}=\log 2
$$




\section{DDAMULIRA}

and

$$
\log B_{2}=\max \left\{h\left(\gamma_{2}\right), \frac{\left|\log \gamma_{2}\right|}{2}, \frac{1}{2}\right\}=\frac{1}{2}
$$

Thus,

$$
b^{\prime}=\frac{\left|k_{2}-k_{1}\right|}{2 \log B_{2}}+\frac{\left|k_{2}\left(n_{1}+m_{1}\right)-k_{1}\left(n_{2}+m_{2}\right)\right|}{2 \log B_{1}} \leq k_{2}+\frac{k_{2}}{\log 2}<3 k_{2} .
$$

Now, Theorem 3.2 tells us that with

$$
\Gamma_{2}=\left(k_{2}-k_{1}\right) \log 2-\left(k_{2}\left(n_{1}+m_{1}\right)-k_{1}\left(n_{2}+m_{2}\right)\right) \log \alpha,
$$

we have

$$
\log \left|\Gamma_{2}\right|>-24.34 \times 2^{4}\left(\max \left\{\log \left(3 k_{2}\right)+0.14,10.5\right\}\right)^{2} \cdot(2 \log 2) \cdot(1 / 2) .
$$

Thus,

$$
\log \left|\Gamma_{2}\right|>-270\left(\max \left\{\log \left(3 k_{2}\right)+0.14,10.5\right\}\right)^{2} .
$$

By comparing the above inequality with (4.15), we get

$$
2 m_{3} \log \alpha-\log \left(24 k_{2}\right)<270\left(\max \left\{\log \left(3 k_{2}\right)+0.14,10.5\right\}\right)^{2} .
$$

If $k_{2} \leq 10523$, then $\log \left(3 k_{2}\right)+0.14<10.5$. Thus, the last inequality above gives

$$
2 m_{3} \log \alpha<270 \times 10.5^{2}+\log (24 \times 10523),
$$

giving $m_{3}<30942$ in this case. Otherwise, $k_{2}>10523$, and we get

$$
2 m_{3} \log \alpha<272\left(1+\log k_{2}\right)^{2}+\log \left(24 k_{2}\right)<280\left(1+\log k_{2}\right)^{2},
$$

which gives

$$
m_{3}<160\left(1+\log k_{2}\right)^{2} .
$$

We have just proved the following lemma.

Lemma 4.2. If $m_{3}>100$, then either

(i) $k_{2} \leq 10523$ and $m_{3}<30942$ or

(ii) $k_{2}>10523$, in which case $m_{3}<160\left(1+\log k_{2}\right)^{2}$.

Now suppose that some $m$ is fixed in (2.1), or at least we have some good upper bounds on it. We rewrite (2.1) using (1.1) and (4.1) as

so

$$
\frac{1}{2}\left(\delta^{k}+\eta^{k}\right)=L_{m}\left(\alpha^{n}+\beta^{n}\right),
$$

$$
\delta^{k}\left(2 L_{m}\right)^{-1} \alpha^{-n}-1=-\frac{1}{2 L_{m}} \eta^{k} \alpha^{-n}+\left(\beta \alpha^{-1}\right)^{n} .
$$

Since $m \geq 1, \beta=-\alpha^{-1}, \eta=\varepsilon \delta^{-1}$ and $\delta^{k}>\alpha^{n+m-1}$, we get

$$
\begin{aligned}
\left|\delta^{k}\left(2 L_{m}\right)^{-1} \alpha^{-n}-1\right| & \leq \frac{1}{2 L_{m} \delta^{k} \alpha^{n}}+\frac{1}{\alpha^{2 n}} \leq \frac{\alpha}{\alpha^{2(n+m)}}+\frac{1}{\alpha^{2 n}} \\
& \leq \frac{\alpha+1}{\alpha^{2 n}}<\frac{6}{\alpha^{2 n}},
\end{aligned}
$$

where we have used the fact that $n \geq m \geq 0$ and $\alpha+1<6$. Hence,

$$
\left|\Lambda_{3}\right|=\left|\delta^{k}\left(2 L_{m}\right)^{-1} \alpha^{-n}-1\right|<\frac{6}{\alpha^{2 n}} .
$$


We assume that $n_{3}>100$. In particular, $\frac{6}{\alpha^{2 n}}<\frac{1}{2}$ for $n \in\left\{n_{1}, n_{2}\right\}$, so we get by the previous argument that

$$
\left|\Gamma_{3}\right|=\left|k \log \delta-\log \left(2 L_{m}\right)-n \log \alpha\right|<\frac{12}{\alpha^{2 n}} .
$$

We are now set to apply Theorem 3.1 on the left-hand side of (4.16) with the data

$$
t=3, \quad \gamma_{1}=\delta, \quad \gamma_{2}=2 L_{m}, \quad \gamma_{3}=\alpha, \quad b_{1}=k, \quad b_{2}=-1, \quad b_{3}=-n .
$$

First, we need to check that $\Lambda_{3}:=\delta^{k}\left(2 L_{m}\right)^{-1} \alpha^{-n}-1 \neq 0$. If not, then $\delta^{k}=2 L_{m} \alpha^{m}$. The left side belongs to the field $\mathbb{Q}(\sqrt{d})$ but not rational, whereas the right side belongs to the field $\mathbb{Q}(\sqrt{5})$. This is not possible unless $d=5$. In this last case, $\delta$ is a unit in $\mathbb{Q}(\sqrt{5})$ whereas $2 L_{m}$ is not a unit in $\mathbb{Q}(\sqrt{5})$ since the norm of this first element is $4 L_{m}^{2} \neq \pm 1$. So, $\Lambda_{3} \neq 0$. Thus, we can apply Theorem 3.1. We have the field $\mathbb{K}=\mathbb{Q}(\sqrt{d}, \sqrt{5})$, which has degree $D \leq 4$. We also have

$$
\begin{aligned}
h\left(\gamma_{2}\right) & =h\left(2 L_{m}\right)=h(2)+h\left(L_{m}\right) \\
& \leq \log 2+(m+1) \log \alpha<2+m \log \alpha \\
& \leq 2.92 \times 10^{13} \log \delta(1+\log (2 n)) \quad \text { by }(4.12) .
\end{aligned}
$$

So, we take

$$
h\left(\gamma_{1}\right)=\frac{1}{2} \log \delta, \quad h\left(\gamma_{2}\right)=2.92 \times 10^{13} \log \delta(1+\log (2 n)), \quad \text { and } \quad h\left(\gamma_{3}\right)=\frac{1}{2} \log \alpha .
$$

Then,

$$
A_{1}=2 \log \delta, \quad A_{2}=1.18 \times 10^{14} \log \delta(1+\log (2 n)), \quad \text { and } \quad A_{3}=2 \log \alpha .
$$

Then, by Theorem 3.1 we get

$$
\begin{aligned}
\log \left|\Lambda_{3}\right|> & -1.4 \times 30^{6} \times 3^{4.5} \times 4^{2}(1+\log 4)(1+\log n)(2 \log \delta) \\
& \times\left(1.18 \times 10^{14} \log \delta(1+\log (2 n))\right)(2 \log \alpha) \\
> & -8.6 \times 10^{26}(1+\log (2 n))^{2}(\log \delta)^{2} \log \alpha .
\end{aligned}
$$

Comparing the above inequality with (4.16), we get

$$
2 n \log \alpha-\log 6<8.6 \times 10^{26}(1+\log (2 n))^{2}(\log \delta)^{2} \log \alpha,
$$

which implies that

$$
n<4.3 \times 10^{26}(1+\log (2 n))^{2}(\log \delta)^{2} .
$$

We state what we have proved.

Lemma 4.3. If $x_{k}=L_{n} L_{m}$ with $n \geq m \geq 1$, then we have

$$
n<4.3 \times 10^{26}(1+\log (2 n))^{2}(\log \delta)^{2} .
$$

Note that we did not use the assumption that $m_{3}>100$ or that $n_{3}>100$ for Lemma 4.3 because we worked with the inequality (4.16), not with the inequality (4.17). We now assume that $n_{3}>100$ and, in particular, (4.17) holds for $(k, n, m)=\left(k_{i}, n_{i}, m_{i}\right)$ for both $i=1,2$. By the previous procedure, we also eliminate the term involving $\log \delta$ as follows:

$$
\left|k_{2} \log \left(2 L_{m_{1}}\right)-k_{1} \log \left(2 L_{m_{2}}\right)-\left(k_{2} n_{1}-k_{1} n_{2}\right) \log \alpha\right|<\frac{12 k_{2}}{\alpha^{2 n_{1}}}+\frac{12 k_{1}}{\alpha^{2 n_{2}}}<\frac{24 k_{2}}{\alpha^{2 n_{3}}} .
$$

We assume that $\alpha^{2 n_{3}}>48 k_{2}$. If we put

$$
\Gamma_{4}=k_{2} \log \left(2 L_{m_{1}}\right)-k_{1} \log \left(2 L_{m_{2}}\right)-\left(k_{2} n_{1}-k_{1} n_{2}\right) \log \alpha,
$$




\section{DDAMULIRA}

we have that $\left|\Gamma_{4}\right|<1 / 2$. Then, we get that

$$
\left|\Lambda_{4}\right|=\left|e^{\Gamma_{4}}-1\right|<2\left|\Gamma_{4}\right|<\frac{48 k_{2}}{\alpha^{2 n_{3}}} .
$$

We apply Theorem 3.1 to

$$
\Lambda_{4}=\left(2 L_{m_{1}}\right)^{k_{2}}\left(2 L_{m_{2}}\right)^{-k_{1}} \alpha^{-\left(k_{2} n_{1}-k_{1} n_{2}\right)}-1 .
$$

First, we need to check that $\Lambda_{4} \neq 0$. If it were, then it would follow that

$$
\frac{L_{m_{1}}^{k_{2}}}{L_{m_{2}}^{k_{1}}}=2^{k_{1}-k_{2}} \alpha^{k_{2} n_{1}-k_{1} n_{2}} .
$$

We consider the following lemma.

Lemma 4.4. The equation (4.21) has only many small positive integer solutions $\left(k_{i}, n_{i}, m_{i}\right)$ for $i=\{1,2\}$ with $k_{1}<k_{2}$ and $m_{1} \leq m_{2} \leq 6$. Futhermore, none of these solutions lead to a valid solution to the original Diophantine equation (2.1).

Proof. We suppose that (4.21) holds and assume that $\operatorname{gcd}\left(k_{1}, k_{2}\right)=1$. Since $\alpha^{k_{2} n_{1}-k_{1} n_{2}} \in \mathbb{Q}$, it follows $k_{2} n_{1}=k_{1} n_{2}$. Thus, if one of the $n_{1}$ or $n_{2}$ is zero, so is the other. Since $n_{i} \geq m_{i}$ for $i \in\{1,2\}$, it follows that $n_{1}=n_{2}=0, m_{1}=m_{2}=0$, so $x_{k_{1}}=x_{k_{2}}$. Therefore, $k_{1}=k_{2}$, a contradiction. Thus, $n_{1}$ and $n_{2}$ are both positive integers. Next, $L_{m_{1}}^{k_{2}} / L_{m_{2}}^{k_{1}}=2^{k_{1}-k_{2}}<1$. Thus, $L_{m_{1}}^{k_{2}}<L_{m_{2}}^{k_{1}}<L_{m_{2}}^{k_{2}}$, so $L_{m_{1}}<L_{m_{2}}$. This implies that either $\left(m_{1}, m_{2}\right)=(1,0)$ or $m_{1}<m_{2}$. The case $\left(m_{1}, m_{2}\right)=(1,0)$ gives $1 / 2^{k_{1}}=2^{k_{1}-k_{2}}$. Thus, $k_{2}=2 k_{1}$ and since $\operatorname{gcd}\left(k_{1}, k_{2}\right)=1$, we get $k_{1}=1, k_{2}=2$, so $n_{2}=2 n_{1}$. But then $x_{2}=x_{k_{2}}=L_{n_{2}} L_{m_{2}}=L_{2 n_{1}} L_{0}=2 L_{2 n_{1}}$ is even, a contradiction because $x_{2}=2 x_{1} \pm 1$ (by Example 3.6 (i)) is odd. Thus, $m_{1}<m_{2}$. If $m_{2}>6$, the Carmichael Primitive Divisor Theorem for Lucas numbers shows that $L_{m_{2}}$ is divisible by a prime $p>7$ which does not divide $L_{m_{1}}$. This is impossible because it contradicts the assumption that (4.21) holds. Thus, $m_{2} \leq 6$. Furthermore, because $L_{m_{1}}^{k_{2}} / L_{m_{2}}^{k_{1}}=1 / 2^{k_{2}-k_{1}}$, it follows that $L_{m_{1}}^{k_{1}}\left|L_{m_{1}}^{k_{2}}\right| L_{m_{2}}^{k_{1}}$, so $L_{m_{1}} \mid L_{m_{2}}$. So, there are three cases that we analyze:

Case 1. $m_{1}=0, m_{2} \in\{3,6\}$. If $\left(m_{1}, m_{2}\right)=(0,3)$, then $2^{k_{2}} / 4^{k_{1}}=1 / 2^{2 k_{1}-k_{2}}=1 / 2^{k_{2}-k_{1}}$. This gives $2 k_{2}=3 k_{1}$ and because $k_{1}$ and $k_{2}$ are coprime, it follows that $k_{1}=2$ and $k_{2}=3$. Then $x_{2}=x_{k_{1}}=L_{n_{1}} L_{m_{1}}=L_{n_{1}} L_{0}=2 L_{n_{1}}$ is even, a contradiction because $x_{2}=2 x_{1} \pm 1$ is odd. If $\left(m_{1}, m_{2}\right)=(0,6)$, then $2^{k_{2}} / 18^{k_{1}}=1 / 2^{k_{2}-k_{1}}$, which is impossible because examining the exponent of 3 we would get $k_{1}=0$, a contradiction.

Case 2. $m_{1}=2$ and $L_{m_{2}}$ is a power of 2. The case $m_{2}=0$ has been treated so the only other case left is $m_{2}=3$. In this case, $1 / 4^{k_{1}}=1 / 2^{k_{2}-k_{1}}$, giving $k_{2}=3 k_{1}$. Thus, since $\operatorname{gcd}\left(k_{1}, k_{2}\right)=1, k_{1}=1$, and $k_{2}=3$. Since $k_{2} n_{1}=k_{1} n_{2}$, we get $n_{2}=3 n_{1}$. Thus, $x_{1}=L_{n_{1}} L_{1}=L_{n_{1}}$ and $x_{3}=L_{3 n_{1}} L_{3}=4 L_{3 n_{1}}$. Now, $x_{3}=x_{1}\left(4 x_{1}^{2} \pm 3\right)$ (by Example 3.6 (ii)) and the second factor is odd, so the power of 2 dividing $4 L_{3 n_{1}}$ divides $x_{1}=L_{n_{1}}$. But, $4 L_{3 n_{1}}$ is a multiple of 8 because $L_{3 n_{1}}$ is even. Thus, $8 \mid L_{n_{1}}$, which is false.

Case 3. $m_{1}=2$ and $m_{2}=6$. We get $3^{k_{2}} /\left(2.3^{2}\right)^{k_{1}}=1 / 2^{k_{2}-k_{1}}$. Examining the exponent of 3 , we get $k_{2}=2 k_{1}$ and examining the exponent of 2 we also get $k_{2}=2 k_{1}$, so $k_{1}=1$ and $k_{2}=2$. Also, $n_{2}=2 n_{1}$. Thus, $x_{1}=L_{n_{1}} L_{m_{1}}=3 L_{n_{1}}$ and $x_{2}=L_{n_{2}} L_{m_{2}}=18 L_{2 n_{1}}$ is even, a contradiction with the fact that $x_{2}=2 x_{1}^{2} \pm 1$ is odd.

So, by Lemma 4.4, we have $\Lambda_{4} \neq 0$. Thus, we can now apply Theorem 3.1 with the data

$$
\begin{gathered}
t=3, \quad \gamma_{1}=2 L_{m_{1}}, \quad \gamma_{2}=2 L_{m_{2}}, \quad \gamma_{3}=\alpha, \quad b_{1}=k_{2}, \\
b_{2}=-k_{1}, \quad b_{3}=-\left(k_{2} n_{1}-k_{1} n_{2}\right) .
\end{gathered}
$$


We have $\mathbb{K}=\mathbb{Q}(\sqrt{5})$ which has degree $D=2$. Also, using (4.5), we can take $B=4 n_{4}^{2}$. We can also take $A_{1}=2\left(2+m_{1} \log \alpha\right) \leq 4 m_{1} \log \alpha, A_{2}=2\left(2+m_{2} \log \alpha\right) \leq 4 m_{2} \log \alpha$ and $A_{3}=\log \alpha$. Theorem 3.1 gives that

$$
\begin{aligned}
\log \left|\Lambda_{4}\right| & >-1.4 \times 30^{6} \times 3^{4.5} \times 2^{2}(1+\log 2)\left(1+\log \left(4 n_{4}^{2}\right)\right)\left(4 m_{1} \log \alpha\right)\left(4 m_{2} \log \alpha\right) \log \alpha, \\
& >-3.44 \times 10^{12} m_{1} m_{2}\left(1+\log \left(2 n_{4}\right)\right) .
\end{aligned}
$$

By comparing this with the inequality (4.20), we get

$$
2 n_{3} \log \alpha-\log \left(48 k_{2}\right)<3.44 \times 10^{12} m_{1} m_{2}\left(1+\log \left(2 n_{4}\right)\right) .
$$

Since $k_{2}<4 n_{4}$ and $n_{4}>10$, we get that $\log \left(48 k_{2}\right)<2\left(1+\log \left(2 n_{4}\right)\right)$. Thus,

$$
n_{3}<3.58 \times 10^{12} m_{1} m_{2}\left(1+\log \left(2 n_{4}\right)\right) .
$$

All this was done under the assumption that $\alpha^{2 n_{3}}>48 k_{2}$. But if that inequality fails, then

$$
n_{3}<c_{1} \log \left(48 k_{2}\right)<12\left(1+\log \left(2 n_{4}\right)\right)
$$

which is much better than (4.22). Thus, (4.22) holds in all cases. We have just proved the following lemma.

Lemma 4.5. Assuming that $n_{3}>100$, then we have

$$
n_{3}<3.58 \times 10^{12} m_{1} m_{2}\left(1+\log \left(2 n_{4}\right)\right) .
$$

We now start finding effective bounds for our variables.

Case 1. $m_{4} \leq 100$.

Then $m_{1}<100$ and $m_{2}<100$. By Lemma 4.5, we get that

$$
n_{3}<3.58 \times 10^{16}\left(1+\log \left(2 n_{4}\right)\right) .
$$

By Lemma 4.1, we get

$$
\log \delta<4 n_{3} \log \alpha<6.89 \times 10^{16}\left(1+\log \left(2 n_{4}\right)\right) .
$$

By the inequality (4.4), we have that

$$
\begin{aligned}
n_{4} & \leq n_{4}+m_{4}-1 \\
& <k_{2} c_{1} \log \delta \\
& <1.72 \times 10^{27} c_{1}\left(1+\log \left(2 n_{4}\right)\right)^{2}(\log \delta)^{3} \quad(\text { by }(4.5) \text { and Lemma 4.3) } \\
& <\frac{1}{\log \alpha}\left(1.72 \times 10^{27}\left(1+\log \left(2 n_{4}\right)\right)^{2}\right)\left(6.89 \times 10^{16}\left(1+\log \left(2 n_{4}\right)\right)\right)^{3} \\
& <1.17 \times 10^{78} \log \left(1+\log \left(2 n_{4}\right)\right)^{5} .
\end{aligned}
$$

With the help of Mathematica, we get that $n_{4}<4.6 \times 10^{89}$. Thus, using (4.5), we get

$$
\max \left\{k_{2}, n_{4}\right\}<4.6 \times 10^{89} .
$$

We record what we have proved.

Lemma 4.6. If $m_{4}=\max \left\{m_{1}, m_{2}\right\} \leq 100$, then

$$
\max \left\{k_{2}, n_{4}\right\}<4.6 \times 10^{89} .
$$

Case 2. $m_{4}>100$.

Note that $m_{3} \leq 100$ or $m_{3}>100$ in which case, by Lemma 4.2 and the inequality (4.5), we have $m_{3} \leq 160\left(1+\log \left(4 n_{4}\right)\right)^{2}$, provided that $m_{4}>10000$, which we now assume. 


\section{DDAMULIRA}

We let $i \in\{1,2\}$ be such that $m_{i}=m_{3}$ and $j$ be such that $\{i, j\}=\{1,2\}$. We assume that $n_{3}>100$. We work with (4.17) for $i$ and (4.10) for $j$ and noting the conditions $n_{i}>100$ and $m_{j}=m_{4}>100$ are fullfilled. That is,

$$
\begin{aligned}
\left|k_{i} \log \delta+\log \left(2 L_{m_{i}}\right)-n_{i} \log \alpha\right| & <\frac{12}{\alpha^{2 n_{i}}}, \\
\left|k_{j} \log \delta-\log 2-\left(n_{j}+m_{j}\right) \log \alpha\right| & <\frac{12}{\alpha^{2 m_{j}}} .
\end{aligned}
$$

By a similar procedure as before, we eliminate the term involving $\log \delta$. We multiply the first inequality by $k_{j}$, the second inequality by $k_{i}$, subtract the resulting inequalities, and apply the triangle inequalty to get

$$
\begin{aligned}
\left|k_{j} \log \left(2 L_{m_{i}}\right)-k_{i} \log 2-\left(k_{j} n_{i}-k_{i}\left(n_{j}+m_{j}\right)\right) \log \alpha\right| & <\frac{12 k_{j}}{\alpha^{2 m_{i}}}+\frac{12 k_{i}}{\alpha^{2 l_{j}}} \\
& <\frac{24 k_{2}}{\alpha^{2 \min \left\{n_{i}, m_{j}\right\}}} .
\end{aligned}
$$

Assume that $\alpha^{2 \min \left\{n_{i}, m_{j}\right\}}>48 k_{2}$. We put

$$
\Gamma_{5}=k_{j} \log \left(2 L_{m_{i}}\right)-k_{i} \log 2-\left(k_{j} n_{i}-k_{i}\left(n_{j}+m_{j}\right)\right) \log \alpha .
$$

We can write $\Lambda_{5}=\left(2 L_{m_{i}}\right)^{k_{j}} 2^{-k_{i}} \alpha^{\left(k_{j} n_{i}-k_{i}\left(n_{j}+m_{j}\right)\right)}-1$. Under the above assumption and using (4.23), we get that

$$
\left|\Lambda_{5}\right|=\left|e^{\Gamma_{5}}-1\right|<2\left|\Gamma_{5}\right|<\frac{48 k_{2}}{\alpha^{2} \min \left\{n_{i}, m_{j}\right\}} .
$$

We are now set to apply Theorem 3.1 on $\Lambda_{5}$. First, we need to check that $\Lambda_{5} \neq 0$. If it were, then we would get that

$$
L_{m_{i}}^{k_{j}}=2^{k_{i}-k_{j}} \alpha^{\left(k_{j} n_{i}-k_{i}\left(n_{j}+m_{j}\right)\right)} .
$$

We consider the following lemma.

Lemma 4.7. The equation (4.25) has only many small positive integer solutions $\left(k_{i}, k_{j}, n_{i}, n_{j}, m_{i}, m_{j}\right)$ for $i, j=\{1,2\}$ with $k_{1}<k_{2}$ and $m_{1} \leq m_{2} \leq 6$. Futhermore, none of these solutions lead to a valid solution to the original Diophantine equation (2.1).

Proof. Suppose that (4.25) holds and assume that $\operatorname{gcd}\left(k_{1}, k_{2}\right)=1$. Since $\alpha^{\left(k_{j} n_{i}-k_{i}\left(n_{j}+m_{j}\right)\right)} \in \mathbb{Q}$, $k_{j} n_{i}=k_{i}\left(n_{j}+m_{j}\right)$. Next, $L_{m_{i}}^{k_{j}}=2^{k_{i}-k_{j}}$. Thus, $k_{i} \geq k_{j}$, so $i=2, j=1, k_{2}>k_{1}$, and $m_{2} \neq 1$. Because $L_{m_{2}}>1$ is a power of 2 , it follows that $m_{2} \in\{0,3\}$. Suppose $m_{2}=0$. Then, $L_{m_{2}}^{k_{1}}=2^{k_{1}}=2^{k_{2}-k_{1}}$, so $k_{2}=2 k_{1}$. Hence, $k_{1}=1$ and $k_{2}=2$. Further, $n_{2}=2\left(n_{1}+m_{1}\right)$. Thus, $x_{2}=x_{k_{2}}=L_{n_{2}} L_{m_{2}}=2 L_{2\left(n_{1}+m_{1}\right)}$ is even, which false because $x_{2}=2 x_{1}^{2} \pm 1$ is odd. Next, suppose $m_{2}=3$. Then, $4^{k_{1}}=2^{k_{2}-k_{1}}$. Thus, $k_{2}=3 k_{1}$, so $k_{1}=1$ and $k_{2}=3$. Next, $n_{2}=3\left(n_{1}+m_{1}\right)$. Hence, $x_{1}=x_{k_{1}}=L_{n_{1}} L_{m_{1}}$ and $x_{3}=x_{k_{2}}=L_{n_{2}} L_{m_{2}}=4 L_{3\left(n_{1}+m_{1}\right)}$. By the previous argument in the proof of Lemma $4.4,8$ divides $x_{3}=x_{1}\left(4 x_{1}^{2} \pm 1\right)$, so $8 \mid x_{1}$. Since $x_{1}=L_{n_{1}} L_{m_{1}}$ and $8 \nmid L_{n}$ for any $n$, it follows that $L_{n_{1}}$ and $L_{m_{1}}$ are both even. Thus, $3 \mid n_{1}$, $3 \mid m_{1}$. Furthermore, one of $L_{n_{1}}, L_{m_{1}}$ is a multiple of 4 , so one of $n_{1}, m_{1}$ is odd. Suppose both are odd. Then $4\left|L_{n_{1}}, 4\right| L_{m_{1}}$ so $16\left|x_{1}\right| x_{3} \mid 4 L_{3\left(n_{1}+m_{1}\right)}$. This implies that $4 \mid L_{3\left(n_{1}+m_{1}\right)}$, which is false because $3\left(n_{1}+m_{1}\right)$ is an even multiple of 3 , and $2 \| L_{6 m}$ for any $m$. Now, suppose that one of $n_{1}, m_{1}$ is an even multiple of 3 , and the other is odd. Then $\operatorname{ord}_{2}\left(x_{1}\right)=3$, where $\operatorname{ord}_{2}(x)$ is the exponent at which 2 appears in the factorization of $x$. Hence,

$$
3=\operatorname{ord}_{2}\left(x_{3}\right)=\operatorname{ord}_{2}\left(4 L_{3\left(n_{1}+m_{1}\right)}\right)=2+\operatorname{ord}_{2}\left(L_{3\left(n_{1}+m_{1}\right)}\right),
$$


giving $\operatorname{ord}_{2}\left(L_{3\left(n_{1}+m_{1}\right)}\right)=1$. This is again false because $3\left(n_{1}+m_{1}\right)$ is an odd multiple 3 , so it is a number of the form $3+6 m$, and for such numbers we have $4 \| L_{3+6 m}$. Hence, in all instances we have obtained a contradiction.

Thus, by Lemma 4.7, we have that $1 \Lambda_{5} \neq 0$. So, we can apply Theorem 3.1 with the data

$$
\begin{gathered}
t=3, \quad \gamma_{1}=2 L_{m_{i}}, \quad \gamma_{2}=2 \quad \gamma_{3}=\alpha \quad b_{1}=k_{j}, \\
b_{2}=-k_{i}, \quad b_{3}=-\left(k_{j} n_{i}-k_{i}\left(n_{j}+m_{j}\right)\right) .
\end{gathered}
$$

From the previous calculations, we know that $\mathbb{K}=\mathbb{Q}(\sqrt{2})$ which has degree $D=2$ and $A_{1}=4 m_{i} \log \alpha, A_{2}=2 \log 2$ and $A_{3}=\log \alpha$. We also take $B=4 n_{4}^{2}$. By Theorem 3.1, we get that

$$
\begin{aligned}
\log \left|\Lambda_{5}\right| & >-1.4 \times 30^{6} \times 3^{4.5} \times 2^{2}(1+\log 2)\left(1+\log \left(4 n_{4}^{2}\right)\right)\left(4 m_{i} \log \alpha\right)(2 \log 2) \log \alpha, \\
& >-5.18 \times 10^{12} m_{i}\left(1+\log \left(2 n_{4}\right)\right) .
\end{aligned}
$$

Comparing the above inequality with (4.24), we get

$$
2 \min \left\{n_{i}, m_{j}\right\} \log \alpha-\log \left(48 k_{2}\right)<5.12 \times 10^{12} m_{i}\left(1+\log \left(2 n_{4}\right)\right) .
$$

Since $m_{4}>100$, we get using $(4.5)\left(k_{2}<4 n_{4}\right)$ that,

$$
\min \left\{n_{i}, n_{j}\right\}<5.38 \times 10^{12}\left(160\left(1+\log \left(4 n_{4}\right)\right)^{2}\right)\left(1+\log \left(2 n_{4}\right)\right)+\frac{c_{1}}{2} \log \left(192 n_{4}\right),
$$

which implies that

$$
\min \left\{n_{i}, m_{j}\right\}<1.72 \times 10^{15}\left(1+\log \left(2 n_{4}\right)\right)^{3} .
$$

All this was under the assumptions that $n_{4}>10000$, and that $\alpha^{2 \min \left\{n_{i}, m_{j}\right\}}>48 k_{2}$. But, still under the condition that $n_{4}>10000$, if $\alpha^{2 \min \left\{n_{i}, m_{j}\right\}}<48 k_{2}$, then we get an inequality for $\min \left\{n_{i}, n_{j}\right\}$ which is even much better than (4.26). So, (4.26) holds provided that $n_{4}>10000$. Suppose say that $\min \left\{n_{i}, m_{j}\right\}=m_{j}$. Then we get that

$$
m_{3}<160\left(1+\log \left(4 n_{4}\right)\right)^{2}, \quad m_{4}<1.72 \times 10^{15}\left(1+\log \left(2 n_{4}\right)\right)^{3} .
$$

By Lemma 4.5, since $m_{3}>100$, we get

$$
\begin{aligned}
n_{3}< & \left(3.58 \times 10^{12}\right)\left(160\left(1+\log \left(4 n_{4}\right)\right)^{2}\right)\left(1+\log \left(2 n_{4}\right)\right) \\
& \times 1.72 \times 10^{15}\left(1+\log \left(2 n_{4}\right)\right)^{3} \\
< & 1.98 \times 10^{30}\left(1+\log \left(2 n_{4}\right)\right)^{6} .
\end{aligned}
$$

With Lemma 4.1, we get

$$
\log \delta<3.80 \times 10^{30}\left(1+\log \left(2 n_{4}\right)\right)^{6},
$$

which with Lemma 4.3 gives

$$
n_{4}<4.30 \times 10^{26}\left(1+\log \left(2 n_{4}\right)\right)^{2}\left(3.80 \times 10^{30}\left(1+\log \left(2 n_{4}\right)\right)^{6}\right)^{2},
$$

which implies that

$$
n_{4}<6.21 \times 10^{87}\left(1+\log \left(2 n_{4}\right)\right)^{14} .
$$

With the help of Mathematica, we obtain $n_{4}<1.30 \times 10^{122}$. This was proved under the assumption that $n_{4}>10000$, but the situation $n_{4} \leq 10000$ already provides a better bound than $n_{4}<1.30 \times 10^{122}$. Hence,

$$
\max \left\{k_{2}, n_{1}, n_{2}\right\}<1.30 \times 10^{122} .
$$




\section{DDAMULIRA}

This was when $m_{j}=\min \left\{n_{i}, m_{j}\right\}$. Now, we assume that $n_{i}=\min \left\{n_{i}, m_{j}\right\}$. Then, we get

$$
n_{i}<1.72 \times 10^{15}\left(1+\log \left(2 n_{4}\right)\right)^{3} .
$$

By Lemma 4.1, we get that

$$
\log \delta<3.31 \times 10^{15}\left(1+\log \left(2 n_{4}\right)\right)^{3} .
$$

Now by Lemma 4.3 , with Lemma 4.1 to bound $l_{4}$, give

$$
\begin{aligned}
n_{4} & \left.<4.30 \times 10^{26}\left(1+\log \left(2 n_{4}\right)\right)\right)^{2}\left(3.31 \times 10^{15}\left(1+\log \left(2 n_{4}\right)\right)^{3}\right)^{2} \\
& <4.72 \times 10^{57}\left(1+\log \left(2 n_{4}\right)\right)^{10} .
\end{aligned}
$$

This gives $n_{4}<2.44 \times 10^{80}$, which is a better bound than $1.30 \times 10^{122}$. We state what we have proved.

Lemma 4.8. If $m_{4}=\max \left\{m_{1}, m_{2}\right\}>100$ and $n_{3}=\min \left\{n_{1}, n_{2}\right\}>100$, then

$$
\max \left\{k_{2}, n_{1}, n_{2}\right\}<1.30 \times 10^{122} .
$$

It now remains the case when $m_{4}>100$ and $n_{3} \leq 100$. But then, by Lemma 4.1, we get $\log \delta<192$ and now Lemma 4.1 together with Lemma 4.3 give

$$
n_{4}<1.56 \times 10^{31}\left(1+\log \left(2 n_{4}\right)\right)^{2},
$$

which implies that $n_{4}<10^{36}$ and further $\max \left\{k_{1}, n_{1}, n_{2}\right\}<10^{40}$. We record what we have proved.

Lemma 4.9. If $m_{4}>100$ and $n_{3} \leq 100$, then

$$
\max \left\{k_{1}, n_{1}, n_{2}\right\}<10^{40} \text {. }
$$

\section{The Final COMPutations}

5.1. The first reduction. In this subsection we reduce the bounds for $k_{1}, m_{1}, n_{1}$ and $k_{2}, m_{2}, n_{2}$ to cases that can be computationally treated. For this, we return to the inequalities for $\Gamma_{2}, \Gamma_{4}$, and $\Gamma_{5}$.

We return to (4.15), and we set $s=k_{2}-k_{1}$ and $r=k_{2}\left(n_{1}+m_{1}\right)-k_{1}\left(n_{2}+m_{2}\right)$ and divide both sides by $s \log \alpha$ to get

$$
\left|\frac{\log 2}{\log \alpha}-\frac{r}{s}\right|<\frac{24 k_{2}}{\alpha^{2 m_{3}} s \log \alpha} .
$$

We assume that $l_{3}$ is so large that the right side of the inequality in (5.1) is smaller than $1 /\left(2 s^{2}\right)$. This certainly holds if

$$
\alpha^{2 m_{3}}>48 k_{2}^{2} / \log \alpha .
$$

Since $k_{2}<1.3 \times 10^{122}$, it follows that the last inequality (5.2) holds provided that $m_{3} \geq 589$, which we now assume. In this case, $r / s$ is a convergent of the continued fraction of $\tau=\frac{\log 2}{\log \alpha}$ and $s<1.30 \times 10^{122}$. We are now set to apply Lemma 3.3 .

We write $\tau=\left[a_{0} ; a_{1}, a_{2}, a_{3}, \ldots\right]=[1 ; 2,3,1,2,3,2,4,2,1,2,11,2,1,11,1,1,134,2,2, \ldots]$ for the continued fraction expansion of $\tau$ and $p_{k} / q_{k}$ for the $k$-th convergent. We get that $r / s=$ $p_{j} / q_{j}$ for some $j \leq 237$. Furthermore, putting $a(M)=\max \left\{a_{j}: j=0,1, \ldots, 237\right\}$, we get $a(M)=880$. By Lemma 3.3, we get

$$
\frac{1}{882 s^{2}}=\frac{1}{(a(M)+2) s^{2}} \leq\left|\tau-\frac{r}{s}\right|<\frac{24 k_{2}}{\alpha^{2 m_{3}} s \log \alpha},
$$


giving

$$
\alpha^{2 m_{3}}<\frac{882 \times 24 k_{2}^{2}}{\log \alpha}<\frac{882 \times 24 \times\left(1.30 \times 10^{122}\right)^{2}}{\log \alpha},
$$

leading to $m_{3} \leq 1190$. We state what we have just proved.

Lemma 5.1. We have $m_{3}:=\min \left\{m_{1}, m_{2}\right\} \leq 1190$.

If $m_{1}=m_{3}$, then we have $i=1$ and $j=2$, otherwise $m_{2}=m_{3}$ implying that we have $i=2$ and $j=1$. In both cases, the next step is the application of Lemma 3.5 (LLL algorithm) for (4.23), where $n_{i}<1.30 \times 10^{112}$ and $\left|k_{j} n_{i}-k_{i}\left(n_{j}+m_{j}\right)\right|<10^{116}$. For each $m_{j} \in[1,1190]$ and

$$
\Gamma_{5}:=k_{j} \log \left(2 L_{m_{i}}\right)-k_{i} \log 2-\left(k_{j} n_{i}-k_{i}\left(n_{j}+m_{j}\right)\right) \log \alpha,
$$

we apply the LLL algorithm on $\Gamma_{3}$ with the data

$$
\begin{gathered}
t=3, \quad \tau_{1}=\log \left(2 L_{m_{i}}\right), \quad \tau_{2}=\log 2, \quad \tau_{3}=\log \alpha \\
x_{1}=k_{j}, \quad x_{2}=-k_{i}, \quad x_{3}=k_{j} n_{i}-k_{i}\left(n_{j}+m_{j}\right) .
\end{gathered}
$$

Furthermore, we set $X=10^{116}$ as an upper bound to $\left|x_{i}\right|$ for $i=1,2,3$, and $C:=(5 X)^{5}$. A computer search in Mathematica allows us to conclude, with the inequality (4.23), that

$$
2 \times 10^{-480}<\min _{1 \leq \min \left\{n_{i}, m_{j}\right\} \leq 1190}\left|\Gamma_{5}\right|<\frac{24 k_{2}}{\alpha^{2 \min \left\{n_{i}, m_{j}\right\}}} .
$$

Thus, $\min \left\{n_{i}, m_{j}\right\} \leq 1419$. We first assume that $i=1, j=2$. Thus, $n_{1} \leq 1419$ or $m_{j}=$ $\min \left\{n_{i}, m_{j}\right\} \leq 1419$.

Next, we suppose that $m_{j}=\min \left\{n_{i}, m_{j}\right\} \leq 1419$. Since $m_{1}=m_{3} \leq 1190$, we have

$$
m_{3}=\min \left\{m_{1}, m_{2}\right\} \leq 1190 \text { and } m_{4}=\max \left\{m_{1}, m_{2}\right\} \leq 1419 .
$$

Now, returning to the inequality (4.19) which involves

$$
\Gamma_{4}=k_{2} \log \left(2 L_{m_{1}}\right)-k_{1} \log \left(2 L_{m_{2}}\right)-\left(k_{2} n_{1}-k_{1} n_{2}\right) \log \alpha,
$$

we use again the LLL algorithm to estimate the lower bound for $\left|\Gamma_{4}\right|$ and thus, find a bound for $n_{1}$ that is better than the one given in Lemma 4.8. We distinguish the cases $m_{3}<m_{4}$ and $m_{3}=m_{4}$.

5.1.1. The case $m_{3}<m_{4}$. We take $m_{1}=m_{3} \in[1,1190]$ and $m_{2}=m_{4} \in\left[m_{3}+1,1419\right]$ and apply Lemma 3.5 with the data:

$$
\begin{gathered}
t=3, \quad \tau_{1}=2 L_{m_{1}}, \quad \tau_{2}=2 L_{m_{2}}, \quad \tau_{3}=\log \alpha \\
x_{1}=k_{2}, \quad x_{2}=-k_{1}, \quad x_{2}=k_{1} n_{2}-k_{2} n_{1} .
\end{gathered}
$$

We also put $X=10^{116}$ and $C=(20 X)^{9}$. After a computer search in Mathematica, with the inequality (4.19), we confirm that

$$
2 \times 10^{-1120} \leq \min _{\substack{1 \leq m_{3} \leq 1190 \\ m_{3}+1 \leq m_{4} \leq 1419}}\left|\Gamma_{4}\right|<24 k_{2} \alpha^{-2 n_{3}} .
$$

This leads to the inequality

$$
\alpha^{2 n_{3}}<12 \times 10^{1120} k_{2} .
$$

Sustituting for the bound $k_{2}$ given in Lemma 4.8, we get that $n_{1}=n_{3} \leq 2950$. 


\section{DDAMULIRA}

5.1.2. The case $m_{3}=m_{4}$. . In this case $m_{1}=m_{2} \leq 1419$ and we have

$$
\Gamma_{4}=\left(k_{2}-k_{1}\right) \log \left(2 L_{m_{1}}\right)-\left(k_{2} n_{1}-k_{1} n_{2}\right) \log \alpha \neq 0 .
$$

This is similar to the case we have handled in the previous steps and yields the bound on $n_{1}$ which is less than 2950. So in both cases we have $n_{1} \leq 2950$. From the fact that

$$
\log \delta \leq k_{1} \log \delta \leq 4 n_{1} \log \alpha<5678,
$$

and by considering the inequality given in Lemma 4.3, we conclude that

$$
n_{2}<1.4 \times 10^{34}\left(1+\log \left(2 n_{2}\right)\right)^{2},
$$

which with the help of Mathematica, yields $n_{2}<1.12 \times 10^{38}$. We summarize the first cycle of our reductions:

$$
\max \left\{k_{1}, m_{1}\right\} \leq n_{1}<2950 \text { and } \max \left\{k_{2}, m_{2}\right\} \leq n_{2}<1.12 \times 10^{38} .
$$

From (5.6), we note that the upper bound on $n_{2}$ represents a very good reduction of the bound given in Lemma 4.8. Hence, we expect that if we restart our reduction cycle with the new bound on $n_{2}$, then we get better bounds on $n_{1}$ and $n_{2}$. Thus, we return to the inequality (5.1) and take $M=1.12 \times 10^{38}$. A computer seach in Mathematica reveals that

$$
q_{82}>M>n_{2}>k_{2}-k_{1} \text { and } a(M)=\max \left\{a_{i}: 0 \leq i \leq 82\right\}=a_{12}=134,
$$

from which it follows that $m_{3} \leq 100$. We now return to $(5.3)$ and we put $X=1.12 \times 10^{40}$ and $C=(20 X)^{5}$ and then apply the LLL algorithm in Lemma 3.5 to $m_{3} \in[1,100]$. After a computer search in Mathematica, we get

$$
1.04 \times 10^{-139}<\min _{1 \leq m_{3} \leq 100}\left|\Gamma_{4}\right|<24 k_{2} \alpha^{-2 \min \left\{n_{i}, m_{j}\right\}},
$$

then $\min \left\{n_{i}, m_{j}\right\} \leq 410$. By continuing under the assumption that $m_{j}=\min \left\{n_{i}, m_{j}\right\} \leq 426$, we return to $(5.5)$ and put $X=1.12 \times 10^{40}, C=(20 X)^{5}$, and $M=1.12 \times 10^{38}$ for the case $m_{3}<m_{4}$ and the case $m_{3}=m_{4}$. After a computer search, we confirm that

$$
4.39 \times 10^{-168}<\min _{\substack{1 \leq m_{3} \leq 100 \\ m_{3}+1 \leq m_{4} \leq 426}}\left|\Gamma_{4}\right|<24 k_{2} \alpha^{-2 n_{3}} .
$$

This gives $n_{1} \leq 494$, which holds in both cases. Hence, by a similar procedure given in the first cycle, we get that $n_{2}<3 \times 10^{36}$.

We state what we have proved.

Lemma 5.2. Let $\left(k_{i}, n_{i}, m_{i}\right\}$ be a solution to the Diophantine equation $x_{k_{i}}=L_{n_{i}} L_{m_{i}}$, with $0 \leq m_{i} \leq n_{i}$ for $i=1,2$ and $1 \leq k_{1} \leq k_{2}$, then

$$
\max \left\{k_{1}, m_{1}\right\} \leq n_{1} \leq 494 \text { and } \max \left\{k_{2}, m_{2}\right\} \leq n_{2}<3 \times 10^{36} .
$$

5.2. The final reduction. Returning back to (4.9) and (4.17) and using the fact that $\left(x_{1}, y_{1}\right)$ is the smallest positive solution to the Pell equation (1.3), we obtain

$$
\begin{aligned}
x_{k} & =\frac{1}{2}\left(\delta^{k}+\eta^{k}\right)=\frac{1}{2}\left(\left(x_{1}+y_{1} \sqrt{d}\right)^{k}+\left(x_{1}-y_{1} \sqrt{d}\right)^{k}\right) \\
& =\frac{1}{2}\left(\left(x_{1}+\sqrt{x_{1}^{2} \mp 1}\right)^{k}+\left(x_{1}-\sqrt{x_{1}^{2} \mp 1}\right)^{k}\right):=P_{k}^{ \pm}\left(x_{1}\right) .
\end{aligned}
$$

Thus, we return to the Diophantine equation $x_{k_{1}}=L_{n_{1}} L_{m_{1}}$ and consider the equations

$$
P_{k_{1}}^{+}\left(x_{1}\right)=L_{n_{1}} L_{m_{1}} \quad \text { and } \quad P_{k_{1}}^{-}\left(x_{1}\right)=L_{n_{1}} L_{m_{1}},
$$

with $k_{1} \in[1,500], m_{1} \in[0,500]$, and $n_{1} \in\left[m_{1}+1,500\right]$. 
Besides the trivial case $k_{1}=1$, with the help of a computer search in Mathematica on the above equations in (5.8), we list the only nontrivial solutions in Table 1. We also note that

$$
7+5 \sqrt{2}=(1+\sqrt{2})^{3},
$$

so these solutions come from the same Pell equation with $d=2$.

\begin{tabular}{|ccccc|}
\hline \multicolumn{5}{|c|}{$Q_{k_{1}}^{+}\left(x_{1}\right)$} \\
\hline$k_{1}$ & $x_{1}$ & $y_{1}$ & $d$ & $\delta$ \\
\hline 2 & 2 & 1 & 3 & $2+\sqrt{3}$ \\
2 & 5 & 2 & 6 & $5+2 \sqrt{6}$ \\
2 & 10 & 3 & 11 & $10+3 \sqrt{11}$ \\
2 & 4 & 1 & 15 & $4+\sqrt{15}$ \\
2 & 6 & 1 & 35 & $6+\sqrt{35}$ \\
\hline
\end{tabular}

\begin{tabular}{|ccccc|}
\hline \multicolumn{5}{|c|}{$Q_{k_{1}}^{-}\left(x_{1}\right)$} \\
\hline$k_{1}$ & $x_{1}$ & $y_{1}$ & $d$ & $\delta$ \\
\hline 2 & 1 & 1 & 2 & $1+\sqrt{2}$ \\
2 & 2 & 1 & 5 & $2+\sqrt{5}$ \\
2 & 7 & 5 & 2 & $7+5 \sqrt{2}$ \\
2 & 4 & 1 & 17 & $4+\sqrt{17}$ \\
2 & 26 & 1 & 677 & $26+\sqrt{677}$ \\
2 & 179 & 1 & 32042 & $179+\sqrt{32042}$ \\
\hline
\end{tabular}

TABLE 1. Solutions to $P_{k_{1}}^{ \pm}\left(x_{1}\right)=L_{n_{1}} L_{m_{1}}$

From Table 1, we set each $\delta=\delta_{t}$ for $t=1,2, \ldots 10$. We then work on the linear forms in logarithms $\Gamma_{1}$ and $\Gamma_{2}$, in order to reduce the bound on $n_{2}$ given in Lemma 5.2. From the inequality $(4.10)$, for $(k, n, m)=\left(k_{2}, n_{2}, m_{2}\right)$, we write

$$
\left|k_{2} \frac{\log \delta_{t}}{\log \alpha}-\left(n_{2}+m_{2}\right)+\frac{\log 2}{\log \left(\alpha^{-1}\right)}\right|<\left(\frac{12}{\log \alpha}\right) \alpha^{-2 m_{2}},
$$

for $t=1,2, \ldots 10$.

We put

$$
\tau_{t}=\frac{\log \delta_{t}}{\log \alpha}, \quad \mu_{t}=\frac{\log 2}{\log \left(\alpha^{-1}\right)} \quad \text { and } \quad\left(A_{t}, B_{t}\right)=\left(\frac{12}{\log \alpha}, \alpha\right) .
$$

We note that $\tau_{t}$ is transcendental by the Gelfond-Schneider's Theorem and thus, $\tau_{t}$ is irrational. We can rewrite the above inequality, (5.9), as

$$
0<\left|k_{2} \tau_{t}-\left(n_{2}+m_{2}\right)+\mu_{t}\right|<A_{t} B_{t}^{-2 m_{2}}, \quad \text { for } \quad t=1,2, \ldots, 10 .
$$

We take $M=3 \times 10^{36}$ which is the upper bound on $n_{2}$ according to Lemma 5.2 and apply Lemma 3.4 to the inequality (5.10). As before, for each $\tau_{t}$ with $t=1,2, \ldots, 10$, we compute its continued fraction $\left[a_{0}^{(t)}, a_{1}^{(t)}, a_{2}^{(t)}, \ldots\right]$ and its convergents $p_{0}^{(t)} / q_{0}^{(t)}, p_{1}^{(t)} / q_{1}^{(t)}, p_{2}^{(t)} / q_{2}^{(t)}, \ldots$. For each case, by means of a computer search in Mathematica, we find and integer $s_{t}$ such that

$$
q_{s_{t}}^{(t)}>18 \times 10^{36}=6 M \quad \text { and } \quad \varepsilon_{t}=\left\|\mu_{t} q^{(t)}\right\|-M\left\|\tau_{t} q^{(t)}\right\|>0 .
$$

We finally compute all the values of $b_{t}=\left\lfloor\log \left(A_{t} q_{s_{t}}^{(t)} / \epsilon_{t}\right) / \log B_{t}\right\rfloor / 2$. The values of $b_{t}$ correspond to the upper bounds on $m_{2}$, for each $t=1,2, \ldots, 10$, according to Lemma 3.4.

Note that we have a problem at $\delta_{7}=2+\sqrt{5}$. This is because

$$
2+\sqrt{5}=2\left(\frac{1+\sqrt{5}}{2}\right)^{2}=2 \alpha^{2} .
$$

In this case, we have $\Gamma_{1}=\left(k_{2}-1\right) \log 2-\left(n_{2}+m_{2}-2 k_{2}\right) \log \alpha$. Thus,

$$
\left|\frac{\log 2}{\log \alpha}-\frac{n_{2}+m_{2}-2 k_{2}}{k_{2}-1}\right|<\frac{12}{\left(k_{2}-1\right) \alpha^{2 m_{2}} \log \alpha}
$$

FEBRUARY 2020 


\section{DDAMULIRA}

By a similar procedure given in Subsection 5.1 with $M=3 \times 10^{36}$, we get that $q_{77}>M$ and $a(M)=\max \left\{a_{i}: 0 \leq i \leq 77\right\}=134$. From this, we can conclude that $m_{2} \leq 96$.

The results of the computation for each $t$ are recorded in Table 2 .

\begin{tabular}{llcccc}
\hline$t$ & $\delta_{t}$ & $s_{t}$ & $q_{s t}$ & $\varepsilon_{t}>$ & $b_{t}$ \\
\hline 1 & $2+\sqrt{3}$ & 68 & $2.07577 \times 10^{37}$ & 0.319062 & 94 \\
2 & $5+2 \sqrt{6}$ & 91 & $8.19593 \times 10^{37}$ & 0.087591 & 97 \\
3 & $10+3 \sqrt{11}$ & 67 & $2.25831 \times 10^{38}$ & 0.316767 & 96 \\
4 & $4+\sqrt{15}$ & 70 & $2.78896 \times 10^{37}$ & 0.329388 & 94 \\
5 & $6+\sqrt{35}$ & 74 & $1.75745 \times 10^{38}$ & 0.409752 & 96 \\
6 & $1+\sqrt{2}$ & 76 & $2.02409 \times 10^{37}$ & 0.263855 & 94 \\
7 & $2+\sqrt{5}$ & - & - & - & 96 \\
8 & $4+\sqrt{17}$ & 78 & $4.76137 \times 10^{37}$ & 0.131771 & 96 \\
9 & $26+\sqrt{677}$ & 65 & $3.17521 \times 10^{37}$ & 0.356148 & 94 \\
10 & $179+\sqrt{32042}$ & 77 & $3.45317 \times 10^{37}$ & 0.384127 & 94 \\
\hline
\end{tabular}

TABLE 2. First reduction computation results

By replacing $(k, n, m)=\left(k_{2}, n_{2}, m_{2}\right)$ in the inequality (4.17), we can write

$$
\left|k_{2} \frac{\log \delta_{t}}{\log \alpha}-n_{2}+\frac{\log \left(2 L_{m_{2}}\right)}{\log \left(\alpha^{-1}\right)}\right|<\left(\frac{12}{\log \alpha}\right) \alpha^{-2 n_{2}},
$$

for $t=1,2, \ldots, 10$.

We now put

$$
\tau_{t}=\frac{\log \delta_{t}}{\log \alpha}, \quad \mu_{t, m_{2}}=\frac{\log \left(2 L_{m_{2}}\right)}{\log \left(\alpha^{-1}\right)} \quad \text { and } \quad\left(A_{t}, B_{t}\right)=\left(\frac{12}{\log \alpha}, \alpha\right) .
$$

With the above notations, we can rewrite (5.11) as

$$
0<\left|k_{2} \tau_{t}-n_{2}+\mu_{t, m_{2}}\right|<A_{t} B_{t}^{-2 n_{2}}, \quad \text { for } \quad t=1,2, \ldots 10 .
$$

We again apply Lemma 3.4 to the above inequality (5.12), for

$$
t=1,2, \ldots, 10, \quad m_{2}=1,2, \ldots, b_{t}, \quad \text { with } \quad M=3 \times 10^{36} .
$$

We take

$$
\varepsilon_{t, m_{2}}=\left\|\mu_{t} q^{\left(t, m_{2}\right)}\right\|-M\left\|\tau_{t} q^{\left(t, m_{2}\right)}\right\|>0
$$

and

$$
b_{t}=b_{t, m_{2}}=\left\lfloor\log \left(A_{t} q_{s_{t}}^{\left(t, m_{2}\right)} / \epsilon_{t, m_{2}}\right) / \log B_{t}\right\rfloor / 2 .
$$

The case $\delta_{7}=2+\sqrt{5}$ is again treated individually by a similar procedure as in the previous step. With the help of Mathematica, we record the results of the computation in Table 3.

\begin{tabular}{c|cccccccccc}
\hline$t$ & 1 & 2 & 3 & 4 & 5 & 6 & 7 & 8 & 9 & 10 \\
$\varepsilon_{t, m_{2}}>$ & 0.0145 & 0.0002 & 0.0006 & 0.0034 & 0.0106 & 0.0005 & - & 0.0009 & 0.0019 & 0.0010 \\
$b_{t, m_{2}}$ & 97 & 103 & 102 & 99 & 99 & 100 & 102 & 100 & 99 & 100 \\
\hline
\end{tabular}

TABLE 3. Final reduction computation results 
Therefore, $\max \left\{b_{t, m_{2}}: t=1,2, \ldots, 10\right.$ and $\left.m_{2}=1,2, \ldots b_{t}\right\} \leq 103$.

Thus, by Lemma 3.4, we have that $n_{2} \leq 103$ for all $t=1,2, \ldots, 10$. From $\delta^{k} \leq \alpha^{n+m+6}$, we conclude that $k_{1}<k_{2} \leq 198$. Collecting everything together, our problem is reduced to search for the solutions for (2.1) in the following ranges

$$
1 \leq k_{1}<k_{2} \leq 200, \quad 0 \leq m_{1} \leq n_{1} \leq 200, \quad \text { and } \quad 0 \leq m_{2} \leq n_{2} \leq 200 .
$$

After a computer search on the equation (2.1) with the above ranges, we obtained the following solutions, which are the only solutions for the exceptional $d$ cases we have stated in Theorem 2.1:

For the +1 case:

$$
\begin{array}{ll}
(d=3) & x_{1}=2=L_{1} L_{0}, \quad x_{2}=7=L_{4} L_{1} ; \\
(d=15) & x_{1}=4=L_{3} L_{1}=L_{0} L_{0}, \quad x_{5}=15124=L_{11} L_{9} ; \\
(d=35) & x_{1}=6=L_{2} L_{0}, \quad x_{3}=846=L_{8} L_{6} .
\end{array}
$$

For the -1 case:

$$
\begin{array}{ll}
(d=2) & x_{1}=1=L_{3} L_{3}, \quad x_{2}=3=L_{2} L_{1}, \quad x_{3}=7=L_{4} L_{1}, \quad x_{9}=1393=L_{11} L_{4} ; \\
(d=5) & x_{1}=2=L_{1} L_{0}, \quad x_{2}=9=L_{2} L_{2} ; \\
(d=17) & x_{1}=4=L_{3} L_{1}=L_{0} L_{0}, \quad x_{2}=33=L_{5} L_{2} .
\end{array}
$$

This completes the proof of Theorem 2.1.

\section{ACKNOWLEDGEMENTS}

The author thanks F. L., in particular for the contribution to the proofs of Lemma 4.4 and Lemma 4.7. The author was supported by the Austrian Science Fund (FWF) projects: F5510N26 - Part of the special research program (SFB), "Quasi-Monte Carlo Methods: Theory and Applications" and W1230 - "Doctoral Program Discrete Mathematics".

\section{REFERENCES}

[1] A. Baker and H. Davenport, The equations $3 x^{2}-2=y^{2}$ and $8 x^{2}-7=z^{2}$, The Quarterly Journal of Mathematics, 20 (1) (1969), 129-137.

[2] A. Baker and G. Wüstholz, Logarithmic forms and Diophantine geometry, vol. 9, Cambridge University Press, Cambridge, 2008.

[3] Yu. F. Bilu and R. F. Tichy, The Diophantine equation $f(x)=g(y)$, Acta Arithmetica, 95 (3) (2000), 261-288.

[4] E. F. Bravo, C. A. Gómez Ruiz, and F. Luca, On the $x$-coordinates of Pell equations which are sums of two Tribonacci numbers, Periodica Mathematica Hungarica, 77 (1) (2018), 175-190.

[5] Y. Bugeaud, M. Mignotte, and S. Siksek, Classical Classical and modular approaches to exponential Diophantine equations I. Fibonacci and Lucas perfect powers, Annals of Mathematics, 163 (2) (2006), 9691018.

[6] H. Cohen, Number Theory. Volume I: Tools and Diophantine Equations, Graduate Texts in Mathematics 239, Springer, 2007.

[7] M. Ddamulira, On the $x$-coordinates of Pell equations which are products of two Pell numbers, preprint, 2019. https://arxiv.org/abs/1906.06330

[8] M. Ddamulira. On the $x$-coordinates of Pell equations which are sums of two Padovan numbers, preprint, 2019. https://arxiv.org/abs/1905.11322

[9] M. Ddamulira and F. Luca, On the $x$-coordinates of Pell equations which are k-generalized Fibonacci numbers, Journal Number Theory, 207 (2020), 156-195.

[10] A. Dujella and A. Pethö, A generalization of a theorem of Baker and Davenport, The Quarterly Journal of Mathematics, 49 (195) (1998), 291-306.

FEBRUARY 2020 


\section{DDAMULIRA}

[11] B. Kafle, F. Luca, A. Montejano, L. Szalay, and A. Togbé, On the x-coordinates of Pell equations which are products of two Fibonacci numbers, Journal Number Theory, 203 (2019), 310-333.

[12] B. Kafle, F. Luca, and A. Togbé, On the x-coordinates of Pell equations which are Fibonacci numbers II, Colloquium Mathematicum, 149 (1) (2017), 75-85.

[13] B. Kafle, F. Luca, and A. Togbé, $x$-Coordinates of Pell equations which are Tribonacci numbers II, Periodica Mathematica Hungarica, 79 (2) (2019), 157-167.

[14] B. Kafle, F. Luca, and A. Togbé. x-Coordinates of Pell equations which are Lucas numbers, Boletín de la Sociedad Matemática Mexicana, 25 (3) (2019), 481-493.

[15] M. Laurent, M. Mignotte, and Yu. Nesterenko, Formes linéaires en deux logarithmes et déterminants d'interpolation, Journal Number Theory, 55 (2) (1995), 285-321.

[16] F. Luca, A. Montejano, L. Szalay, and A. Togbé, On the $x$-coordinates of Pell equations which are Tribonacci numbers, Acta Arithmetica, 179 (1) (2017), 25-35.

[17] F. Luca and A. Togbé, On the $x$-coordinates of Pell equations which are Fibonacci numbers, Mathematica Scandinavica, 122 (1) (2018), 18-30.

[18] E. M. Matveev, An explicit lower bound for a homogeneous rational linear form in the logarithms of algebraic numbers II, Izv. Ross. Akad. Nauk Ser. Mat., 64 (6) (2000), 125-180, In Russian. English translation in Izvestiya: Mathematics, 64 (6) (2000), 1217-1269.

[19] OEIS Foundation Inc. (2019), The On-Line Encyclopedia of Integer Sequences, https://oeis.org/ A000032.

[20] S. S. Rihane, M. O. Hernane, and A. Togbé, The x-coordinates of Pell equations and Padovan numbers, Turkish Journal of Mathematics, 43 (1) (2019), 207-223.

MSC2010: 11B39, 11D45, 11D61, 11J86.

Institute of Analysis and Number Theory, Graz University of Technology, Kopernikusgasse 24/II, A-8010 Graz, Austria

E-mail address: mddamulira@tugraz.at; mahadi@aims.edu.gh 